\title{
Clay nanocomposites based on poly(vinylidene fluoride-co-hexafluoropropylene): structure and properties
}

Antonios Kelarakis, Suren Hayrapetyan, Seema Ansari, Jason Fang, Luis Estevez, Emmanuel P. Giannelis ${ }^{*}$

Department of Materials Science and Engineering, Cornell University, Ithaca, NY 14853

* To whom correspondence should be addressed. Email: epg2@cornell.edu

Keywords; nanocomposite, clay, toughness, polymorphism

\begin{abstract}
The introduction of organically modified clays to poly(vinylidene fluoride-cohexafluoropropylene) matrix promotes an $\alpha$ to $\beta$ transformation of the crystalline phase in a manner that critically depends upon the nature of the clay surface modifier. In addition, the presence of nanoclay facilitates an energy dissipation mechanism that gives rise to extensive enhancements in mechanical toughness. At ambient and elevated temperatures the dielectric permittivity of nanocomposites dramatically increases compared to the neat polymer. The rheological, mechanical and dielectric properties of hybrids directly reflect the morphological and structural changes induced by clays.
\end{abstract}




\section{Introduction}

The nanoscopic origin of the macroscopic properties in polymer clay nanocomposites has been the subject of intense investigations which have been summarized in a number of reviews [1-9]. One of the key points to the development of this class of hybrid materials is the realization that the native inorganic ions within clay cavities can be replaced by bulkier ammonium ions linked to hydrocarbon chains. This pretreatment can significantly alter the thermodynamics of host/guest interactions, facilitating polymer intercalation within the clay galleries and giving rise to intercalated or exfoliated mesostructures. Clay hybrids often exhibit attractive performance enhancements with respect to thermomechanical properties, dimensional stability, barrier characteristics and flame retardancy. Suffice to say that those enhance properties carry a great promise for a wide range of technological applications.

Conceptually, a number of structural changes induced by nanoparticles can be expressed in terms of mobility confinement of the polymer chains in the vicinity of the organic/inorganic interface. In that sense, clay nanocomposites can serve as model systems to study polymer segmental dynamics under severe confinement as in the case of ultrathin films or fluids in restricted environments [10]. The interfacial structure and dynamics determine, in turn, the macroscopic properties of the nanocomposites so that performance improvements can be directly related to the dispersion state of the filler, the total area of the internal interfaces and to the strength of matrix-nanoparticle interactions.

At the same time, due to their rigid nature, clay platelets can be viewed as nucleating agents that are able to modify the crystallization behavior of the polymer matrix [11]. However, martensiticlike transformations of the crystalline phase have been observed only in selected polymer-clay combinations. The most well explored examples of this type are the development of $\gamma$ phase in Nylon 6 at the expense of the $\alpha$ phase in the neat polymer [12,13] and the evolution of $\beta$ phase instead of the $\alpha$ form in PVDF [14-24]. The latter has a considerable impact in applications where pyroelectric, piezoelectric, ferroelectric or magnetostrictive response is desirable [25], given that the all-trans configuration of $\beta$ phase imparts a high dipole moment $\left(7.010^{-30} \mathrm{Cm}\right.$ /repeat unit) due to the alignment of the polar C-F bonds perpendicular to the polymer axis chain. 
In this study, we expand our previous efforts by considering a series of clay nanocomposites based on Poly(vinylidene fluoride-co-hexafluoropropylene). The introduction of defect modifications to PVDF backbone can generate copolymers that exhibit certain advantages compared to homopolymer such as enhanced piezoelectric properties and improved mechanical behavior [26-29]. PVDF based materials are considered ideal candidates for several applications varying from electroacoustic and electromechanical convertors, actuators, ferroelectric memory devices to mechatronics and artificial muscles. Here, we attempt to assess the role of clay surface modification on the crystallization behavior of the polymer matrix, the morphology of the nanocomposite and the macroscopic response of the hybrids materials with respect to their rheological, mechanical and dielectric properties.

\section{Experimental}

\section{Materials}

Poly(vinylidene fluoride-co-hexafluoropropylene) referred hereafter as PVDF-HFP is a random copolymer (6\% HFP comonomer) purchased from Aldrich in pellet form and was ground to fine powder before further use.

Three commercial organically modified montmorillonites (MMT) clays were used; I.30T from Nanocor Inc., which is octadecyltrimethyl ammoniun-substituted MMT (MMT-Alk), Cloisite 30B from Southern Clay Products, a bis(hydroxyethyl)methyl tallow ammoniun-exchanged MMT (MMT-OH), and Perchem 97 from Southern Clay Products that is modified with benzyltallowdimethylammonium (MMT-Ar). The unmodified montmorillonite (MMT-Na ${ }^{+}$) was provided by Southern Clay Products. All clays were repeatedly washed in ethanol-water to remove any excess ammonium ions before use.

Prior to nanocomposites preparation, both clays and polymer were dried in a vacuum oven for several hours. The components were first thoroughly mixed in a Flack- Tek DAC-150 FV speed mixer, before being melt coextruded by means of a laboratory scale DSM twin screw microcompounder at $215{ }^{\circ} \mathrm{C}$ under flowing nitrogen for $4 \mathrm{~min}$. Specimens with ring or dumbbell shape were prepared by a Daca Instruments microinjector with the barrel at $260{ }^{\circ} \mathrm{C}$, the mold at 
$90{ }^{\circ} \mathrm{C}$ and the injection pressure at $690 \mathrm{kPa}$. For comparison, pure copolymer samples were treated in an identical fashion.

For the co-precipitation method, $5 \mathrm{wt} \%$ PVDF-HFP solution in N,N-dimethyl formamide, (DMF, Sigma Aldrich) was mixed with the appropriate amount of $2 \mathrm{wt} \%$ MMT-Ar in DMF (that had been subjected to vigorous stirring overnight at elevated temperature followed by ultrasonication for $2 \mathrm{~h}$ ). The mixture was then stirred overnight and ultrasonicated for another $2 \mathrm{~h}$. The ternary solution was then coagulated by adding water as antisolvent and the precipitated material was dried under vacuum for 4 days at $70{ }^{\circ} \mathrm{C}$ to form a nanocomposite abbreviated as MMT-Ar/DMF. Specimens were prepared by compression molding at $\mathrm{T}=210{ }^{\circ} \mathrm{C}$ and $20 \mathrm{MPa}$ pressure.

\section{Methods}

Wide-angle, X-ray diffraction (WAXS) spectra were recorded at room temperature using a Scintag Inc. $\theta-\theta$ goniometer ( $\mathrm{CuK \alpha} \alpha$ radiation, $\lambda=1.54 \AA$ ). Transmission electron microscopy (TEM) imaging was performed on FEI Tecnai T12 using microtomed ultrathin samples. Scanning electron microscopy (SEM) images of fracture surfaces were taken on a KECK FESEM, LEO 1550. Differential scanning calorimetry (DSC) thermographs were collected on a TA Instrument Q1000 series calorimeter over the temperature range -50 to $150{ }^{\circ} \mathrm{C}$ at a scan rate of $10{ }^{\circ} \mathrm{C} / \mathrm{min}$. Tensile tests were performed at room temperature with an Instron 5500 Mechanical Tester at constant strain rate of $5 \mathrm{~mm} / \mathrm{min}$.

Rheological measurements were performed on a Paar Physica Modular Compact Rheometer 300 (MCR 300) equipped with parallel plate geometry (diameter $25 \mathrm{~mm}$ ). Measurements were performed in small amplitude oscillatory shear, at a fixed temperature $240{ }^{\circ} \mathrm{C}$, in a dry nitrogen atmosphere to suppress oxidative degradation. The frequency scans covered a range from 0.1 to $600 \mathrm{~s}^{-1}$. Dielectric properties were recorded using a Novacontrol GmbH broadband dielectric spectrometer in the temperature range -100 to $150{ }^{\circ} \mathrm{C}$. 


\section{Results and discussion}

The d-spacing increase in all XRD patterns of the PVDF-HFP nanocomposites compared to the neat clays (Figure 1) provide clear evidence of polymer intercalation in the silicate layers. A representative TEM image is shown in Figure 2 suggesting a uniform dispersion of clay tactoids. The intercalated mesostructures observed for the clay nanocomposites imply the presence of considerable matrix-filler interactions that promotes the development of large organic/inorganic interfaces. Within the set of nanocomposites considered here, the expansion of the d-spacing of the organoclays does not seem to be sensitive to the nature of organic modifier. XRD trace of the nanocomposite prepared by co-precipitation method (MMT-Ar/DMF in Figure 1) shows close similarities with the XRD pattern of the melt-extruded analogue, indicating that melt extrusion does not cause any degradation effects on the organic modifier.

At higher q values, the diffraction pattern of the neat copolymer (Figure 3) can be indexed as the crystal planes of the monoclinic unit cell of the $\alpha$ phase of PVDF [21]. The presence of bulky HFP comonomer units into the homopolymer backbone results in a decrease in crystallinity. Incorporation of organically modified clay nanoparticles to PVDF-HFP matrix leads to the evolution of a new reflection peak $\beta(200) / \beta(110)$ at $2 \theta=21^{\circ}$, characteristic of the orthorhombic unit cell of $\beta$ phase of PVDF. Clearly, the amount of the $\beta$ phase in the nanocomposite depends on the type of clay used with MMT-Alk $<$ MMT-OH $<$ MMT-Ar. The clay induced crystal transformation was further confirmed by FTIR spectroscopy (Figure 4). The $\alpha$-phase peaks centered at 763 and $796 \mathrm{~cm}^{-1}$ are clearly observed only for the neat polymer while the $\beta$-phase peak at $840 \mathrm{~cm}^{-1}$ can only be seen in the nanocomposites. TEM images shown in Figure 5 reveal pronounced morphological variations due to the nucleation efficacy of nanoclays. Interestingly, while the neat polymer forms well defined spherulites with radii in the order of several micrometers, the size of those superstructures drastically decreases in the presence of organosilicates.

The rich polymorphism of PVDF (forming five distinct phases namely $\alpha, \beta, \gamma, \delta, \varepsilon$ ) originates in large from the very similar van der Waals volume of fluorine compared to hydrogen atom that can allow high symmetry and offer flexibility to the polymer chain. The stabilization of the $\beta$ phase of PVDF induced by clay nanoparticles has been originally related to the spatial 
confinement of the polymer chains imposed by the rigid nanofillers [14]. It has been suggested that crystallization of PVDF under constrained conditions favors the evolution of $\beta$ modification, in a manner similar to that observed in binary immiscible blends containing PVDF droplets as the minor component [30]. Alternatively, a mechanism in analogy to that developed for the epitaxial crystallization of $\beta$ modification on $\mathrm{KBr}$ substrate has been proposed [31]. The mechanism highlights the geometrical similarities between the crystal lattice of the inorganic phase (KBr, clay) and the $\beta$ phase of PVDF [17,32]. It should be noted that the $\beta$ modification was also found to be favored in the presence of Ag nanoparticles [33], functionalized multiwall carbon nanotubes [34] and functionalized graphene sheets [35]. The stress induced crystal transformation from $\alpha$ to $\beta$ phase of PVDF, even in the absence of clay, can be manifested from XRD patterns shown in Figure 3 taken across the tensile fractured surface of PVDF-HFP copolymer. The uniaxial or biaxial deformation of PVDF in the temperature range between the glass transition and the melting point of the polymer in the absence or presence of electric field is a widely applied technique for the preparation of the $\beta$ phase of PVDF [36-38].

DSC thermographs (second heating cycle) of various nanocomposites are compared with the neat polymer in Figure 6. The addition of MNT- $\mathrm{Na}^{+}$does not affect the melting point of the polymer $\mathrm{T}_{\mathrm{m}}=157^{\circ} \mathrm{C}$, however dispersion of organically modified clays to the polymer matrix leads to higher $\mathrm{T}_{\mathrm{m}}$. In particular, the melting points of MMT-Alk, MMT-OH and MMT-Ar filled hybrids were found to be 159,161 and $165{ }^{\circ} \mathrm{C}$ respectively. In other words, the displacement of $\mathrm{T}_{\mathrm{m}}$ in nanocomposites scales with the amount of $\beta$ phase, as a direct consequence of the enhanced thermal stability of $\beta$ crystals.

The stress-strain plots shown in Figure 7 indicate the presence of a toughening mechanism induced by nanoclays. In particular, the elongation at break increases from $20 \%$ for the neat copolymer to approximately 110 and 130\% for MMT-OH and MMT-Ar hybrids respectively, without any adverse effect on Young modulus. Moreover, the MMT-Alk nanocomposite exhibits a modest increase in strain to break, while the unmodified MMT hybrid shows the same maximum strain as the unfilled sample. Overall, the fracture toughness of the MMT-Alk, MMT$\mathrm{OH}$ and MMT-Ar nanocomposites exhibit 2,5 and 6 fold increase respectively compared to the 
unfilled counterpart. We note that the trend in increasing toughness is consistent with the amount of $\beta$ phase present.

Dramatic enhancements in toughness have been reported for a limited number of clay based nanocomposite systems including PVDF [17] and PVDF based blends [39]. In general, nanofillers homogeneously dispersed in a polymer matrix, in addition to inducing changes in crystallinity (both degree and type of phase) can give rise to a percolated network that has a protective role against the onset of catastrophic cracking; the load transfer efficiency largely depends upon the interfacial nanofiller/polymer shear stress [40]. The improved elasticity observed here points out to major conformational effects of the macromolecular chains at the clay-matrix interface as well as in the bulk. The SEM image of the tensile fractured cross-section of MMT-Ar hybrid is shown in Figure 8. The SEM image essentially demonstrates the architectural features of a super tough surface that can effectively direct and distribute the load during uniaxial deformation. Specifically, we note the organization of the polymer matrix into fiber-like conformations and superstructures oriented parallel to the stretching direction. In rubbery matrices, the realignment of the clay particles themselves along the stretching direction effectively blocks crack propagation and deflection [41,42].

In an effort to better understand the mechanical response we studied the viscoelastic response of the nanocomposites and the neat polymer using isothermal $\left(T=240{ }^{\circ} \mathrm{C}\right)$ frequency scans (Figure 9). The rheological behavior of the neat polymer corresponds to an entangled polymer melt having short relaxation time. The introduction of MMT- $\mathrm{Na}^{+}$leaves the rheological signature essentially unaffected. In contrast, profound deviations of the ideal melt behavior, mainly in the low frequency limit, can be observed for the organoclay based nanocomposites. This behavior is typical for several classes of hybrid materials and has been attributed to the formation of a physically cross-linked superstructure due to the presence of the nanofillers [43-47]. Essentially, the development of such a physical network tends to increase the relaxation time of the melt, while at high frequencies the viscoelastic spectra of nanocomposites exhibit reduced sensitivity to the presence of nanofillers. This behavior indicates that nanoclays cannot significantly hinder the short-range motions of the polymer matrix, as opposed to the long-range motions. 
The dielectric response for the neat polymer and nanocomposites is shown in Figure 10. Four processes are typically seen for such copolymers [48-50]. A $\beta$ relaxation below $-40{ }^{\circ} \mathrm{C}$ due to local dynamics in the polymer chains, a relaxation around $-40{ }^{\circ} \mathrm{C}$ due to the glass transition of the amorphous phase, a relaxation of the crystalline phase above $0{ }^{\circ} \mathrm{C}$ and an order-disorder transition of the ordered ferroelectric phase at even higher temperatures. The crystalline transition virtually disappears in systems where the polar $\beta$ phase dominates. The dielectric response at low temperatures $\left(0{ }^{\circ} \mathrm{C}\right)$ seems to be insensitive to the presence of the clay. In contrast, the clay affects significantly the response at high temperatures. In addition to those already mentioned, the dielectric response at high temperatures is affected by a number of processes including mobility of ionic carriers, and development of space charges and polarization in the amorphous phase. Upon heating, the combined action of those effects gives rise to a steep increase of dielectric permittivity, even in the absence of clay. This steep increase is more dramatic for the nanocomposites and it commences at much lower temperatures. Interestingly, the increase is consistent with the trend seen before (i.e. nanocomposites with higher $\beta$ phase content exhibit higher values of $\varepsilon \square$, consistent with the increased polarity of the $\beta$ phase).

A remarkable correlation between the structural features and the macroscopic properties of the nanocomposites can be established. Recall that the amount of $\beta$ crystallinity of nanocomposites increases in the order MMT $<$ MMT-Alk $<$ MMT-OH $<$ MMT-Ar. Significantly, the toughness of the (solid) composites, the dielectric permittivity at higher temperatures and the complex viscosity of the melts appear to follow the same trend. While the minimal impact of the MMT$\mathrm{Na}^{+}$clay can be attributed to the limited compatibility with the polymer, all three organoclays exhibit virtually similar levels of dispersability (Figure 1). Therefore, the density and the strength of localized polymer-clay interactions dictate the structural characteristics of the hybrid materials which, in turn, have a profound impact on the extent of reinforcement on various macroscopic properties.

The moderate effects observed for MNT-Alk can be traced back to the interactions of the silicate surface with the matrix, despite the lack of any significant chemical affinity of the aliphatic surfactant with PVDF-HFP. On the other hand, hydrogen bonding between the hydroxyl groups of the organic modifier and the electronegative fluorine of PVDF-HFP can give rise to strong 
polymer-particle interactions. The substantial reinforcement observed for MNT-Ar nanocomposites can be attributed to the synergy of favorable energetics due to noncovalent attraction between the face of aromatic rings with the C-F as well as C-H bonds [51,52]. Note that based on $a b$ initio calculations, the interaction energies for benzene complexes with methane and fluoromethane were estimated -1.5 and $-4.2 \mathrm{kcal} /$ mole, respectively and have been attributed to dispersion (long-range) rather than charge transfer contributions [52].

\section{Conclusions}

Organically modified clays promote the formation of the $\beta$ phase of PVDF-HFP and, by doing so, drastically alter the polarity of the matrix. Moreover, the introduction of clays enables a remarkable toughening mechanism, due to a major reconstruction of the mesoscale features of the polymer. A close correlation between the crystallization behavior of the matrix and several macroscopic properties of the hybrids can be drawn. In the melt state, favorable interactions locally (macromolecular chains- clay modifier) are reflected in the strong viscoelastic response of the hybrids. During crystallization, those interactions can direct more effectively the evolution of $\beta$ phase at the expense of $\alpha$ phase. In the solid state, the clay induced polar $\beta$ phase imparts significant increases in dielectric permittivity to the hybrids, while the fiber-like $\beta$ crystals and the plate-like silicates are the structural units of a network with superior mechanical performance.

\section{Acknowledgements}

E. P. Giannelis acknowledges the support of Award No. KUS-C1-018-02, made by King Abdullah University of Science and Technology (KAUST). 


\section{References}

[1]Giannelis EP. Adv Mater 1996;8: 29-35

[2] Le Baron PC, Wang Z, Pinnavaia TJ. Applied Clay Science 1999;15:11-29

[3] Ray SS, Okamoto M. Progress in Polymer Science 2003; 28:1539-1641

[4] Nguyen QT, Baird DG. Advances in Polymer Technology 2006;25:270-285

[5] Yuan Q, Misra RDK. Materials Science and Technology 2006;22:742-755

[6] Okada A, Usuki A. Macromol Mater Eng 2006;291:1449-1476

[7] Paul DR, Robenson LM. Polymer 2008;49:3187-3204

[8] Pavlidou S, Papaspyrides CD. Progress in Polymer Science 2008;33:1119-1198

[9] Camargo PHC, Satyanarayana KG, Wypch F. Materials Research 2009;12:1-39

[10] Anastasiadis SH, Karatasos K, Vlachos G, Manias E, Giannelis EP. Physical Review Letters 2000;84:915-918

[11] Jog JP. Materials Science and Technology 2006;22:797-806

[12] Medellin-Rodriguez FJ, Burger C, Hsiao BS, Chu B, Vaia R, Phillip S. Polymer 2001; 42: 9015-9023

[13] Maiti P, Okamoto M. Macromol Mater Eng 2003; 288:440-445

[14] Priya L, Jog JP. J Polym Sci, Polym Phys 2002;40:1682-1689

[15] Priya L, Jog JP. J Appl Polym Sci 2003;89:2036-2040

[16] Priya L, Jog JP. J Polym Sci, Polym Phys 2003;41:31-38

[17] Shah D, Maiti P, Gunn E, Schmidt DF, Jiang DD, Batt CA, Giannelis EP. Adv Mater 2004;16:1173-1177

[18] White JL, Kim Y. J Appl Polym Sci 2004;92:1061-1071

[19] Pramoda KP, Mohamed A, Phang IY, Liu T. Polym Int 2005; 54:226-232

[20] Dillon DR, Tenneti KK, Li CY, Ko FK, Sics I, Hsiao BS. Polymer 47 2006;47: 1678-1688

[21] Buckley J, Cebe P, Cherdack D, Crawford J, Ince BS, Jenkins M, Pan J, Reveley M,

Washington N, Wolchover N. Polymer 2006;47:2411-2422

[22] Patro TU, Mhalgi MV, Khakhar DV, Misra A. Polymer 2008;49:3486-3499

[23] Yu W, Zhao Z, Zheng W, Song Y, Li B, Long B, Jiang Q. Materials Letters 2008; 62:747750 
[24] Wu T, Xie T, Yang G. Journal of Polymer Science: Part B: Polymer Physics, 2009; 47:

903-911

[25] Lovinger AJ. Science 1983;220:1115-1121

[26] Neese B, Wang Y, Chu B, Ren K, Liu S, Zhang QM, Huang C, West J. Appl Phys Lett 2007; 90: 242917

[27] Zhou X, Zhao X, Suo Z, Zou C, Runt J, Liu S, Zhang S, Zhang QM. Appl Phys Lett 2009;94:162901

[28] Zhang QM, Bharti V, Zhao X. Science 1998;280:2101-2104

[29] Xu H, Cheng ZY, Olson D, Mai T, Zhang QM, Kavarnos G. Appl Phys Lett 2001;78:23602362

[30] Yang X, Kong X, Tan S, Li G, Ling W, Zhou E. Polym Int 2000;49:1525-1528

[31] Lovinger AJ. Polymer 1981;22:412-413

[32] Yamada E, Nishioka A, Suzuki H, Koda T, Ikeda S. Jpn J Appl Phys 2007;46:7371-7374

[33] Manna S, Batabyal SK, Nandi AK. J Phys Chem B 2006; 110:12318-12326

[34] Manna S, Nandi AK. J Phys Chem C 2007,111,14670-14680

[35] Ansari S, Giannelis EP. J Polym Sci Part B: Polym Phys 2009;47;888-897

[36] Lando JB, Olf HG, Peterlin A. J Polym Sci 1966;4:941-951

[37] McGrath JC, Ward IM. Polymer 1980;21:855-857

[38] Sajkiewicz P, Wasiak A, Goclowski Z. Eur Polym J 1999;35:423-429

[39] Kelarakis A, Giannelis EP, Yoon K. Polymer 2007;48:7567-7572

[40] Kelarakis A, Yoon K, Sics I, Somani RH, Hsiao BS, Chu B. Polymer 2005;46: 5103-5117

[41] Gersappe D. Physical Review Letters 2002; 89; 058301

[42] Shah D, Maiti P, Jiang DD, Batt CA, Giannelis EP. Adv Mat 2005;17:525-528

[43] Xu W, Raychowdhury S, Jiang DD, Retsos H, Giannelis EP. Small 2008;5:662-669

[44] Krishnamoorti R, Giannelis EP. Macromolecules 1997;30:4097-4102

[45] Krishnamoorti R, Yurekli K. Curr Opin Colloid Interface Sci 2001;6:464-470

[46] Kelarakis A, Yoon K, Somani RH, Chen X, Hsiao BS, Chu B. Polymer 2005;46:1159111599

[47] Bhattacharya SN, Kamal MR, Gupta RK In Polymeric Nanocomposites :Theory and

Practice Published by Hanser, Munich, 2007, ch. 4

[48] Cebe T, Grubb DT. Macromolecules 1984; 17:1374-1384 
[49] Kochervinskii VV, Malyshkina IA, Markin GV, Gavrilova ND, Bessonova NP. J Appl Polym Sci 2007,105:1101-1117

[50] Lu Y, Claude J, Norena-Franco LE, Wang Q. J Phys Chem B 2008; 112:10411-10416

[51] Lu YX, Zou JW, Wang YH,Yu QS. Chem. Phys. 2007; 334:1-7

[52] Tsuzuki S, Honda K, Uchimaru T, Mikami M, Tanabe K. J. Phys. Chem. A 2002; 106:44234428 


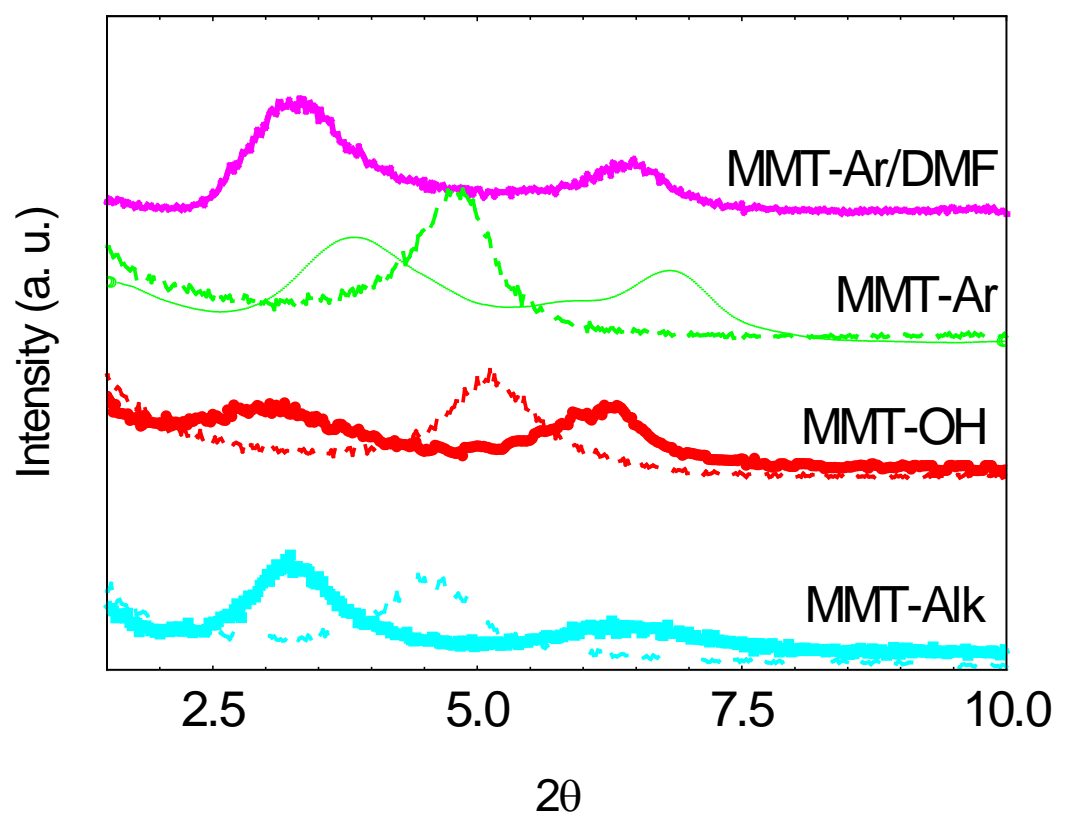

Figure 1. XRD patterns of PVDF-HFP clay nanocomposites (solid lines) compared to the corresponding neat nanoclays (dashed lines). 


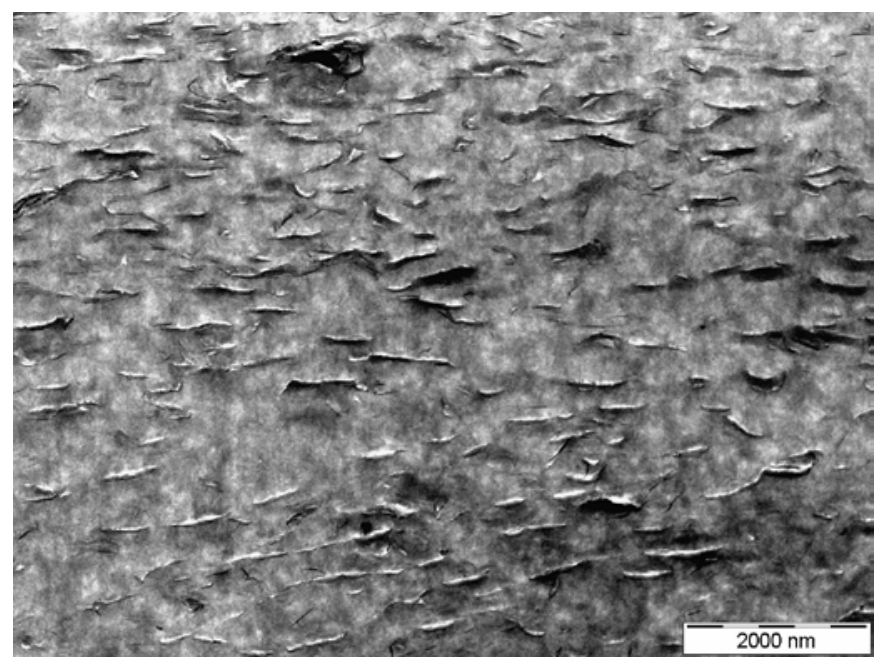

Figure 2. TEM image of a PVDF-HFP based nanocomposite containing 5wt\% MMT-Ar. 


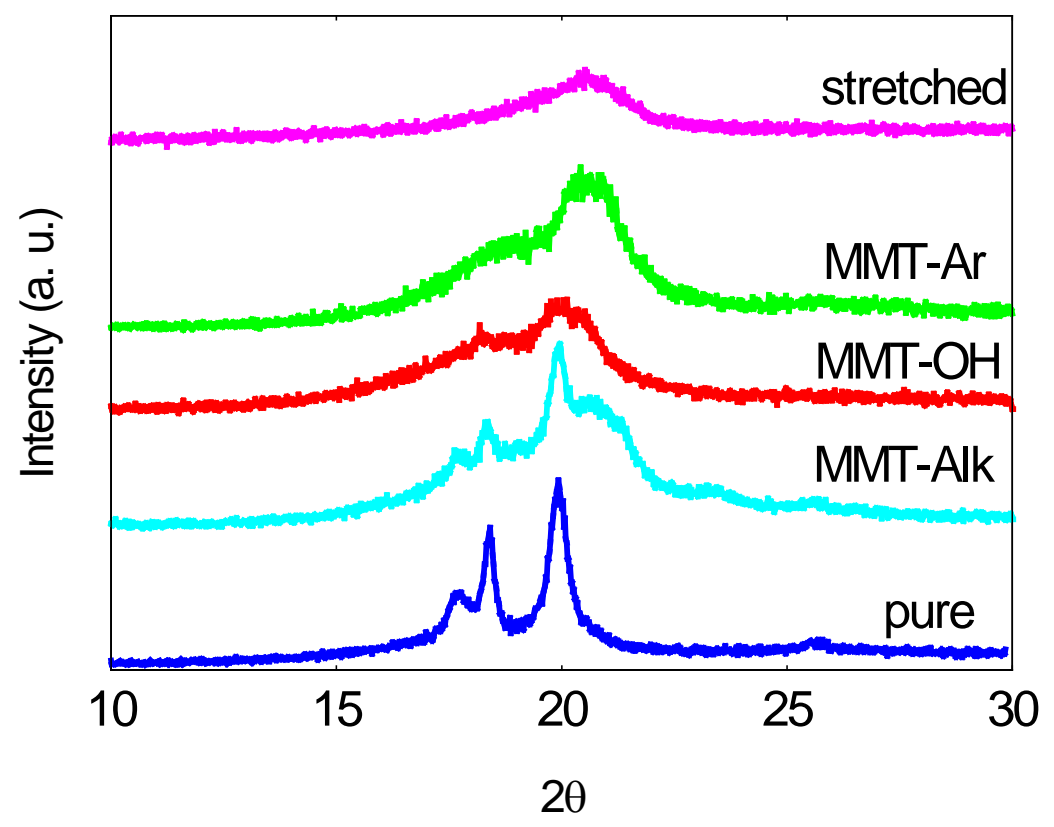

Figure 3. Crystalline reflections of PVDF-HFP and clay based nanocomposites. 


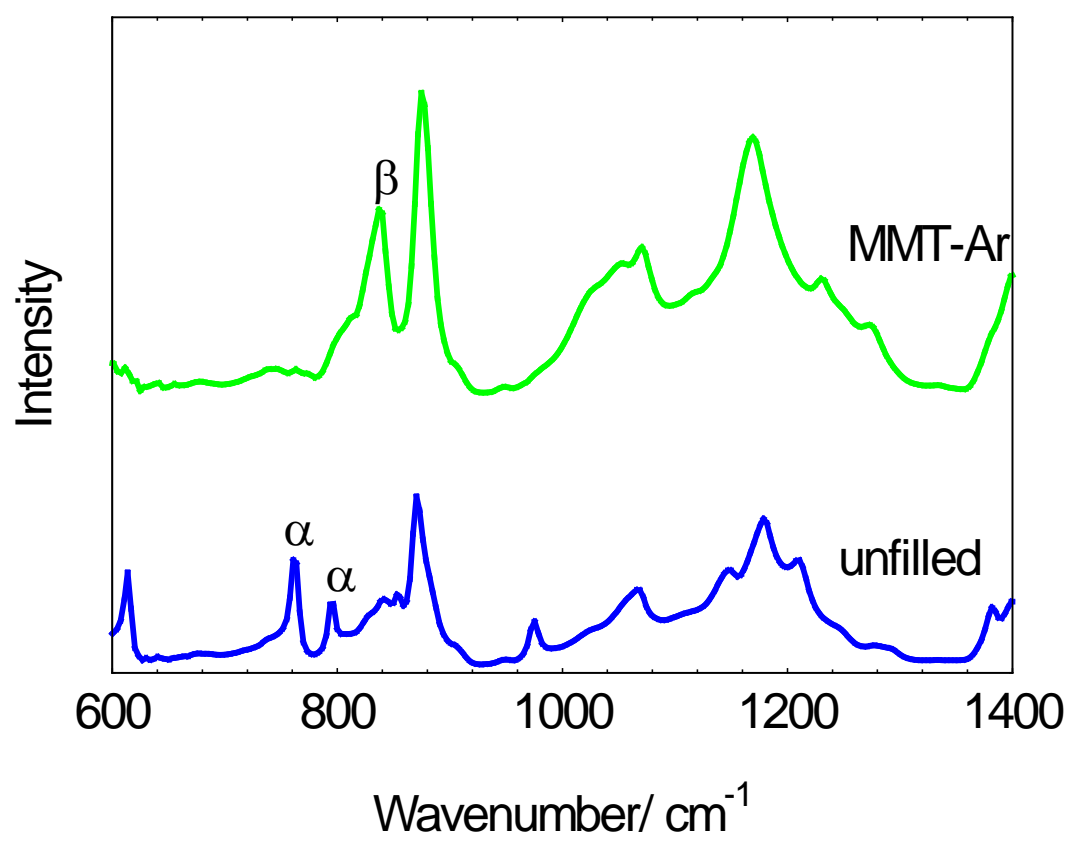

Figure 4. FTIR spectra of neat PVDF-HFP copolymer (bottom) and a nanocomposite containing $5 \mathrm{wt} \%$ MMT-Ar (top). The characteristics frequencies for $\alpha$ and $\beta$ phases are shown in the spectra. 
a

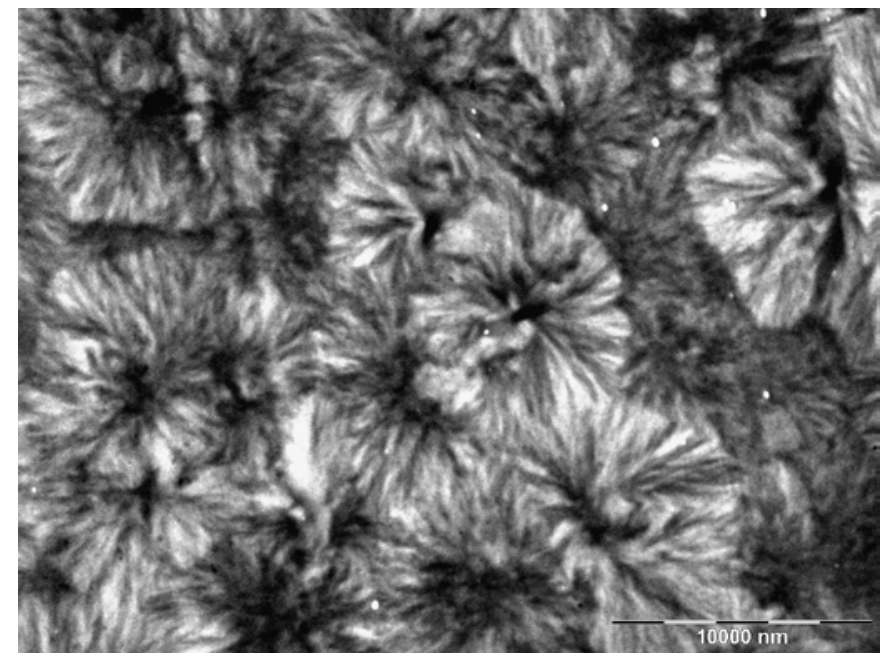

b

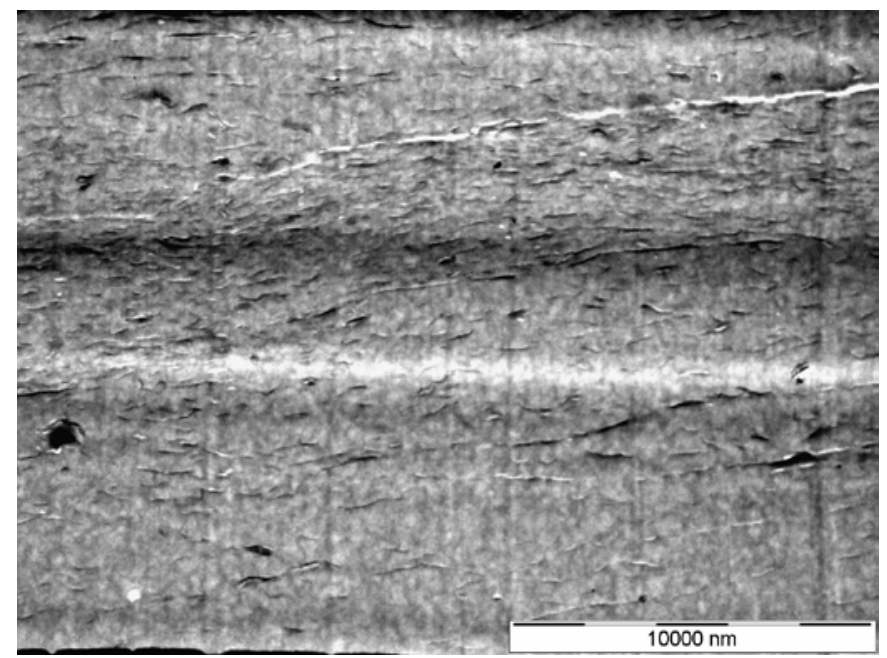

Figure 5. TEM images of a) neat copolymer showing well defined spherulites and b) a 5 wt $\%$ MMT-Ar nanocomposite. 


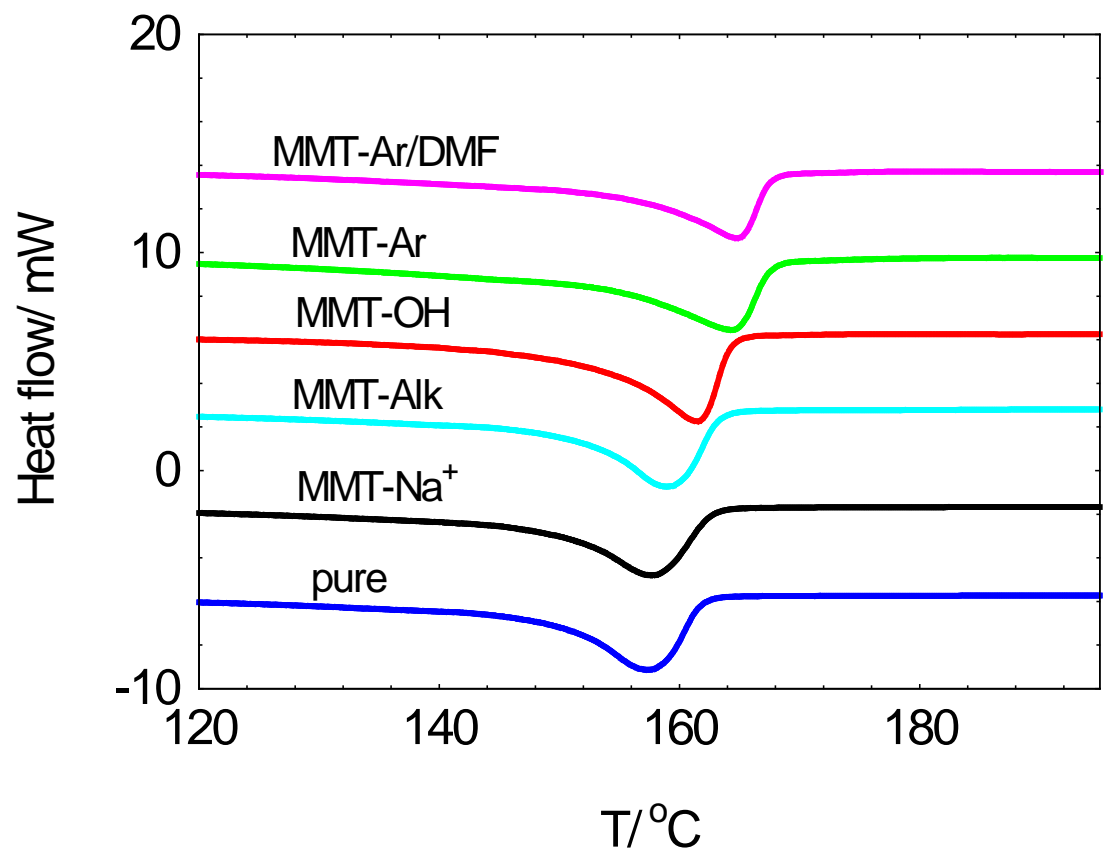

Figure 6. DSC thermographs (second heating cycle) of various PVDF-HFP clay nanocomposites. 


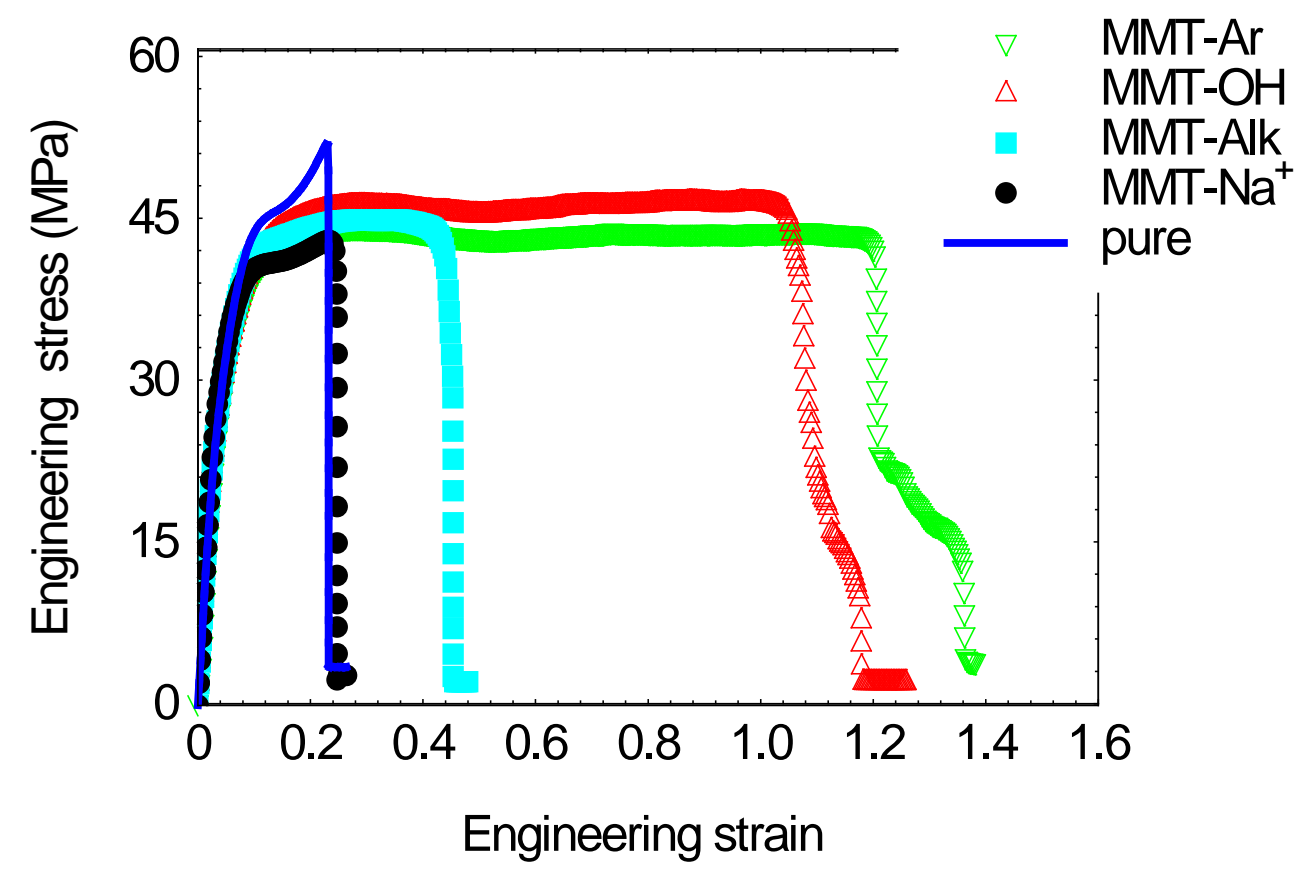

Figure 7. Uniaxial (tensile) deformation plots of PVDF-HFP copolymer and clay nanocomposites performed at room temperature and deformation rate of $5 \mathrm{~mm} / \mathrm{min}$. 


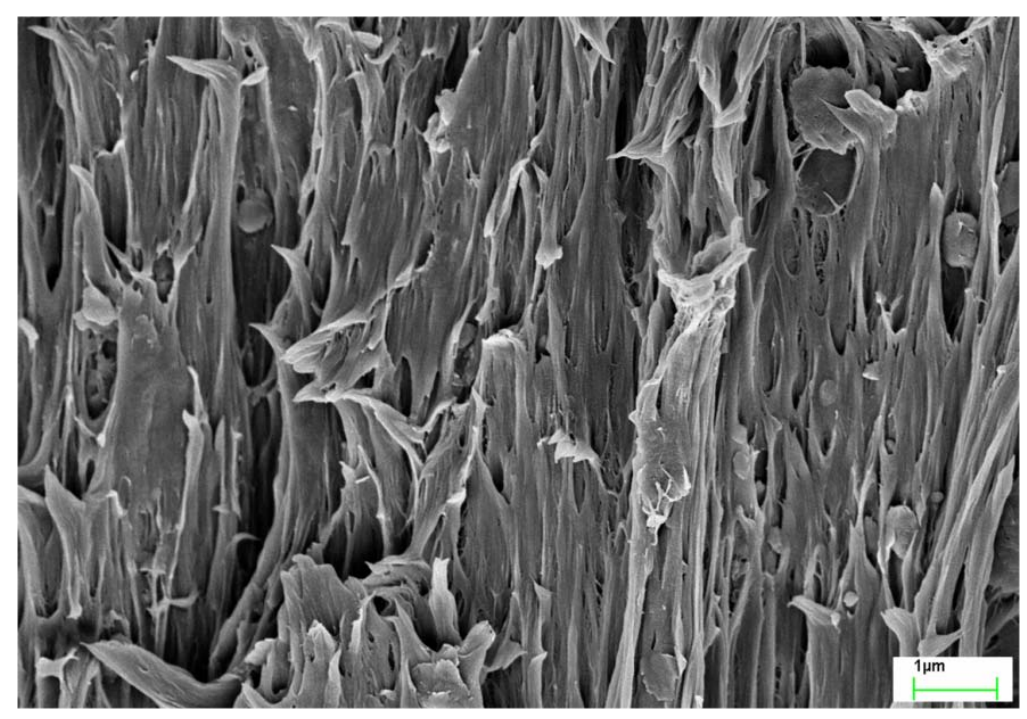

Figure 8. SEM image of the fractured surface of 5wt\% MMT-Ar nanocomposite. 


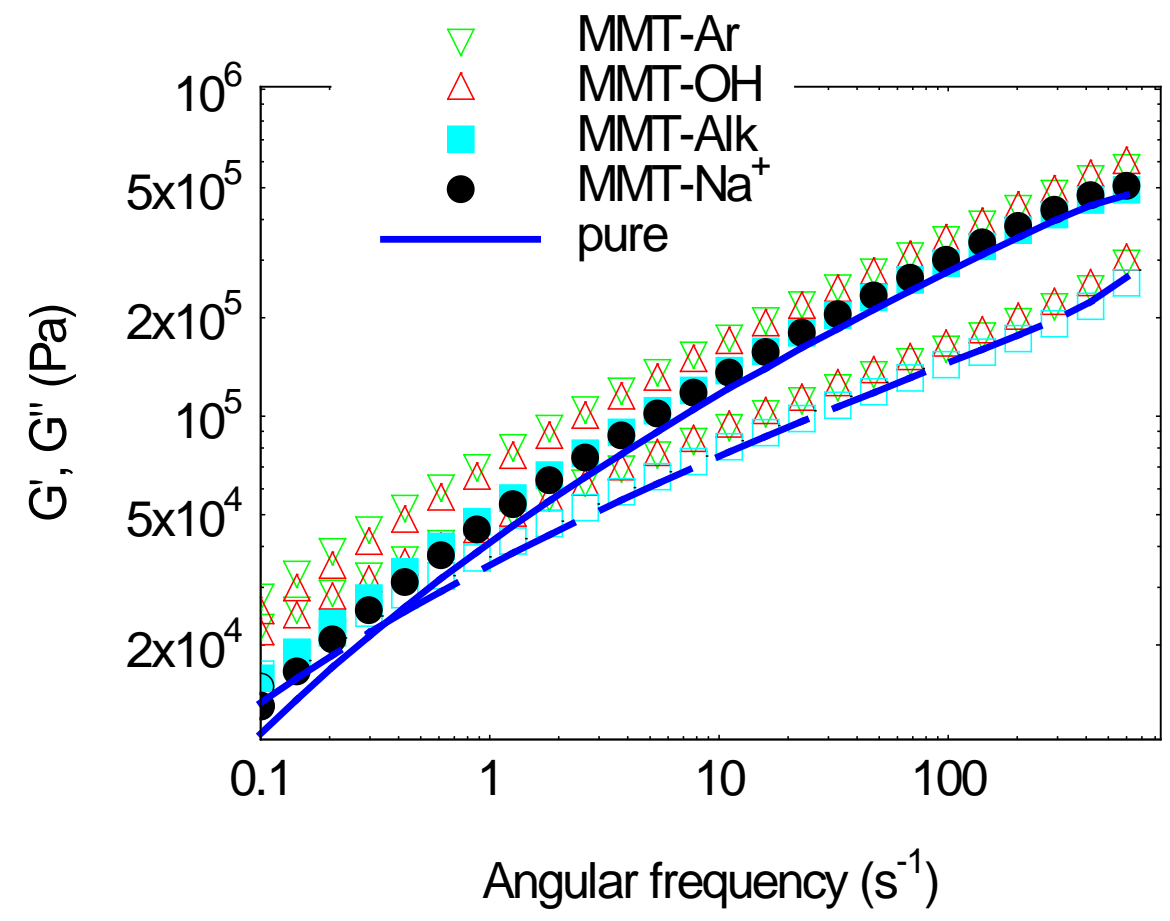

Figure 9. Frequency dependence of storage ( $\left.G^{\prime}\right)$ and loss ( $\left.G^{\prime \prime}\right)$ modulus of PVDF-HFP based nanocomposites containing $5 \mathrm{wt} \%$ clay; $\mathrm{MMT}^{-\mathrm{Na}^{+}}$(circles), MMT-Alk (squares), MMT-OH (upwards triangles), MMT-Ar (downwards triangles). Filled symbols correspond to $\mathrm{G}^{\prime}$ and unfilled symbols to $G^{\prime \prime}$. Solid and dashed line refer to $G^{\prime}$ and $G^{\prime \prime}$ of neat polymer respectively. $\left(\mathrm{T}=240^{\circ} \mathrm{C}\right)$ 


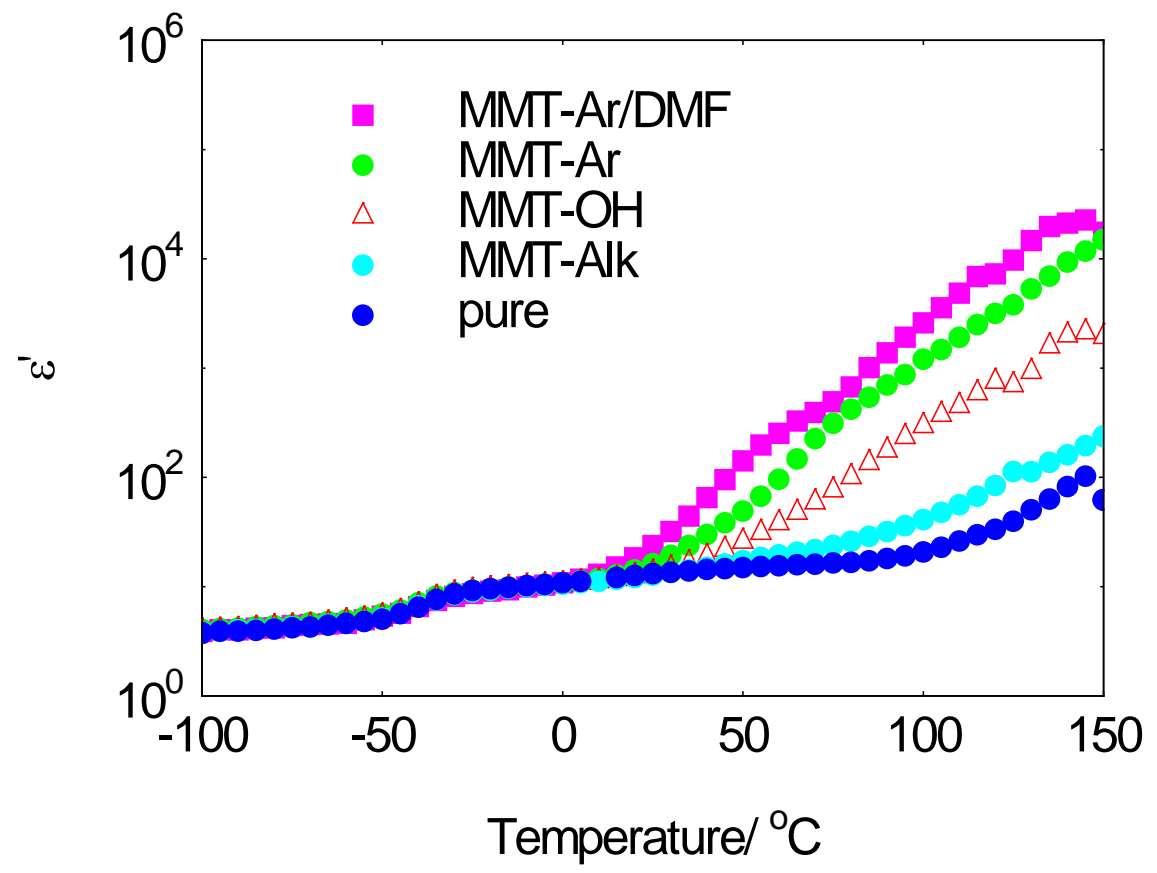

Figure 10. Real part of dielectric permittivity of several PVDF-HFP nanocomposites as a function of temperature at frequency $1 \mathrm{~Hz}$. 


\section{Graphical content entry}

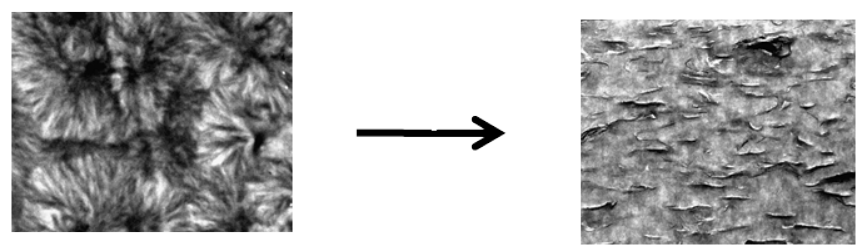

The introduction of organically modified clays to Poly(vinylidene fluoride-cohexafluoropropylene) matrix promotes an $\alpha$ to $\beta$ transformation of the crystalline phase in a manner that critically depends upon the nature of the clay surface modifier. A close correlation between the structural features and the macroscopic properties in a series of clay nanocomposites can be drawn. 